Part of Journal of Research of the National Bureau of Standards, Volume 25, July 1940

\title{
A DUAL BRIDGE FOR THE MEASUREMENT OF SELF IN- DUCTANCE IN TERMS OF RESISTANCE AND TIME
}

\author{
By Harvey L. Curtis and Leon W. Hartman*
}

\section{ABSTRACT}

A dual bridge for the measurement of self inductance in terms of resistance and time is described, its theory is developed, and experimental results are given. The dual bridge consists of a primary bridge and a detector bridge, both of which are independently balanced for continuous current. The primary bridge has the inductance to be measured in one of its resistance arms and a source of unidirectional pulsating current in its battery arm. In its galvanometer arm there is connected, through a rectifying switch, the detector bridge which has an integrating galvanometer in one of its resistance arms and a battery as a source of current. The current from the battery is adjusted until its effect on the integrating galvanometer is equal and opposite to that of the pulses? bridge. The theory not only gives the formula for computing the inductance, but also a formula for determining the maximum number of pulses per second that may be employed with a given set of bridge constants in order to obtain a desired accuracy. The experimental results show that the method can readily be used to measure inductances as large as 1 henry, with an accuracy of 1 part in 1,000. There are indications that results of much higher accuracy can be obtained.

\section{CONTENTS}

II. Theory of the method

III. Experimental test of the method

1. The bridge

2. The galvanometers

3. The batteries

4. The cam-operated switches

5 . Control and measurement of the frequency of the pulsating current

6. Procedure in making observations

7. Results_...

IV. Summary and conclusion

\section{INTRODUCTION}

A method which employs pulsating current in a dual bridge has been developed for the measurement of self inductance in terms of resistance and time. This method, which uses two Wheatstone bridges, each of which is balanced for continuous current, has been applied to the determination of the inductance of several inductors. The distinctive feature is the use of a unidirectional pulsating current for energizing one of the bridges, and the comparison of the time integral of the current in the galvanometer arm of this bridge with the con-

\footnotetext{
*President, University of Nevada.
} 
tinuous current in one of the arms of the other bridge. In this way a null method is employed, using a current with a known wave form.

A method for the measurement of self inductance in terms of resistance and time by means of transient currents was proposed by Maxwell. ${ }^{1}$ The inductor is inserted in one arm of a Wheatstone bridge, the bridge is balanced with continuous current, and the ballistic deflection of the galvanometer is observed when the battery circuit is opened or closed. The inductance is computed from the deflection, sensitivity, period, and damping of the galvanometer, and from the resistances of the bridge arms. Values of inductance obtained by this method usually do not have an accuracy greater than 1 percent.

A method ${ }^{2}$ which has frequently been employed for determining a self inductance in terms of resistance and time requires an intermediary capacitance. The inductance is measured in terms of resistance and capacitance in an alternating-current bridge, then the capacitance is measured in terms of resistance and time, using a pulsating current. If the capacitance has the same value when used in the two bridges, the inductance can be computed from the resistances in the bridges and the fundamental frequency of the pulsating current. The uncertainty in the value of an inductance obtained by this method may be only a few parts in a million.

The dual-bridge method has been devised in order to have available another method for determining the value of a self inductance in terms of resistance and time. The theory has been so developed that the inductance can be computed from the resistances of the bridges, the electromotive forces of the batteries, and the frequency of the pulsating current. All correction terms that would introduce an error of as much as 1 part in a million have been considered. Experimental determinations have been made in order to show the possibilities of the method, though the apparatus available did not permit measurements of the highest accuracy.

\section{THEORYTOF THE METHOD}

The method may be briefly described as a primary bridge having a detector bridge connected in that arm which, in a Wheatstone bridge, contains only a galvanometer. A cam-operated switch opens and closes the current in the primary bridge at regular intervals and a second switch on the same shaft as the first opens and closes the connections from the primary bridge to the detector bridge. In this way a unidirectional pulsating current is produced in the arms of the dual bridge. The average value of the pulsating current in one of the arms of the detector bridge is made equal to a continuous current that is supplied to this arm by a separate battery. There is then no deflection of a ballistic galvanometer which is directly connected in the arm.

A schematic diagram of the dual bridge is shown in figure 1. The current for the primary bridge is supplied by a battery having an electromotive force $E$. The currents which originate in this battery are designated in the various arms as $I$ with an appropriate subscript. The current for the detector bridge is supplied by a battery having an

\footnotetext{
1 Phil. Mag. 155, 475 (1865).

2 For a recent description of this method, see Curtis, Moon, and Sparks, J. Research NBS 16, 1 (1936) RP857.
} 
electromotive force $e$. The currents which originate in this battery are constant and are designated as $i$ with a suitable subscript.

In making a measurement with the dual bridge, each bridge is first balanced with continuous current by connecting it to an auxiliary galvanometer, which is not shown in the drawing. The continuous current for balancing the primary bridge is obtained from battery $E$ by short-circuiting switch $C_{1}$, and for the detector bridge from battery $e$ by disconnecting switch $C_{2}$. The respective bridges are balanced by adjusting the resistances in the $Q$ and $g$ arms. While the detector bridge is being balanced, the coil of the galvanometer in the $g$ arm should be clamped. The resistances of the balanced bridges have the relationships $g k=f h$ and $Q R=P S$. In the next step of the measure-

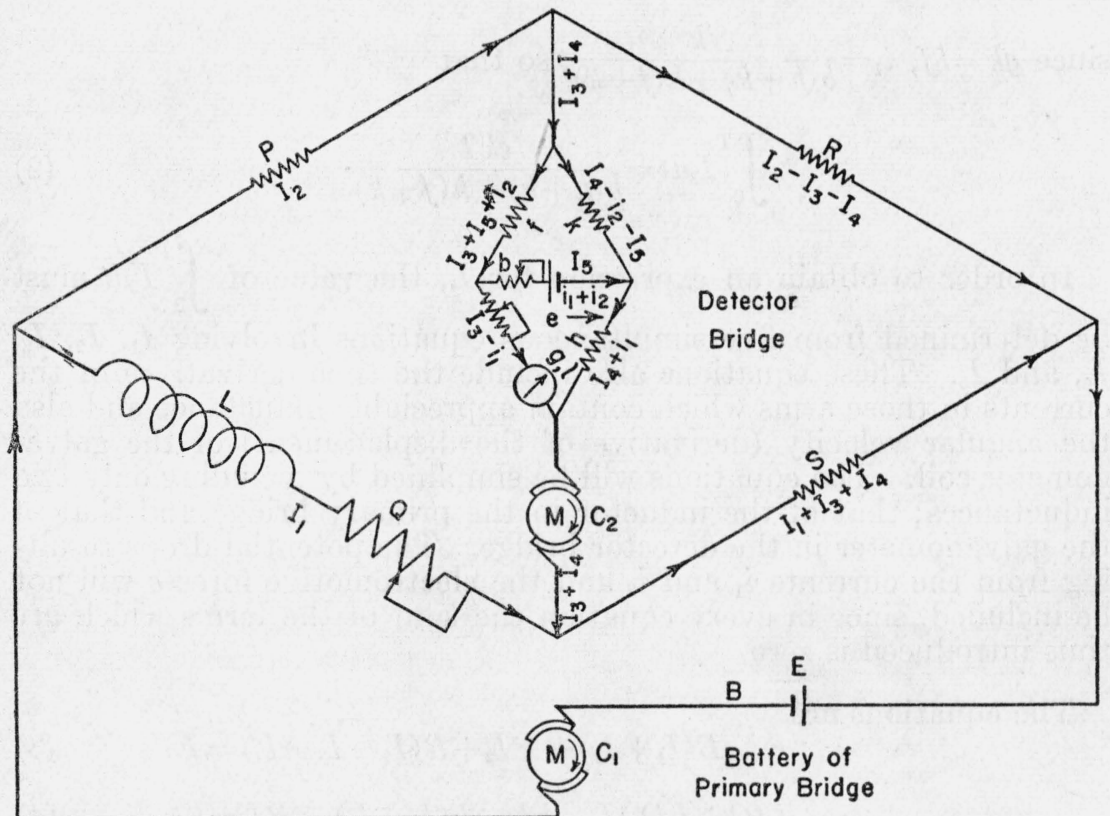

FIGURE 1.-Diagram of the dual bridge for measuring a self inductance in terms of resistance and time.

$$
\text { If } P=R=S \text { and } f=h=k, L=T \cdot \frac{e}{E} \cdot \frac{4(B+P)(P+f)}{b+f} \text {. }
$$

ment, the battery and galvanometer arms of the primary bridge are connected to switches $C_{1}$ and $C_{2}$, respectively, which are attached to a single shaft that rotates with a constant speed. With the switches as shown in figure 1 , switch $C_{1}$ makes and breaks the circuit of the primary bridge, and switch $C_{2}$ keeps the connection between the primary and the detector bridges closed during the time that the current is building up in the primary bridge, but opens this connection before the current starts to die down. By changing the angular position of $C_{2}$ relative to $C_{1}, C_{2}$ can be made to stay closed when the primary current is dying down, and to open before it starts to build up. In either case the operation of these switches produces in the detector bridge a series of pulses having a time interval, $T$. The magnitude of each pulse depends on the inductance $L$, on the electromotive force $E$ of the battery 
in the primary bridge, and on the resistances in the arms of the two bridges. The galvanometer $g$, in the detector bridge has a period which is at least $100 T$, so that the series of pulses produces a deflection with a small periodic component. The average value of this deflection is made zero by adjusting the resistance $b$, which changes the constant current in the detector bridge. Since the average deflection of the ballistic galvanometer is zero, the quantity of electricity sent through it as a result of the pulsating current is the same in any complete period as the quantity resulting from the continuous current. Hence

$$
\int_{0}^{T} I_{3} d t=i_{1} T
$$

since $g k=h f, i_{1}=\frac{e k}{b(h+k)+h(f+k)}$, so that

$$
\int_{0}^{T} I_{3} d t=\frac{e k T}{b(h+k)+h(f+k)}
$$

In order to obtain an expression for $L$, the value of $\int_{0}^{T} I_{3} d t$ must be determined from five simultaneous equations involving $I_{1}, I_{2}, I_{3}$, $I_{4}$, and $I_{5}$. These equations also include the time derivatives of the currents in those arms which contain appreciable inductance and also the angular velocity (derivative of the displacement) of the galvanometer coil. The equations will be simplified by including only two inductances; that of the inductor in the primary bridge and that of the galvanometer in the detector bridge. The potential drops resulting from the currents $i_{1}$ and $i_{2}$ and the electromotive force $e$ will not be included, since in every equation the sum of the terms which are thus introduced is zero.

The equations are

$$
\begin{aligned}
B\left(I_{1}+I_{2}\right)+P I_{2}+R\left(I_{2}-I_{3}-I_{4}\right) & =E . \\
\left(Q+L D_{t}\right) I_{1}-h I_{4}-k\left(I_{4}-I_{5}\right)-P I_{2} & =0 . \\
S\left(I_{1}+I_{3}+I_{4}\right)-R\left(I_{2}-I_{3}-I_{4}\right)+h I_{4}+k\left(I_{4}-I_{5}\right) & =0 . \\
f\left(I_{3}+I_{5}\right)+\left(g+l D_{t}\right) I_{3}-h I_{4}-k\left(I_{4}-I_{5}\right) & =H D_{t} \theta . \\
b I_{5}-k\left(I_{4}-I_{5}\right)+f\left(I_{3}+I_{5}\right) & =0 .
\end{aligned}
$$

The meaning of all the symbols used in these equations is readily understood by reference to figure 1 , except $\theta$, which indicates the deflection of the galvanometer, and $H$, which signifies the electromotive force generated in the galvanometer coil when it is moving with unit angular velocity. These equations are based on the assumption that one can neglect the resistance of the commutator which is in series with the detector bridge. If an accuracy of 1 part in a million is required, and if each of the resistances of the arms of the detector bridge is $10,000 \mathrm{ohms}$ or more, the resistance of the commutator and leads must be less than $0.01 \mathrm{ohm}$. 
Each of the eq 3 to 7 will be multiplied by $d t$ and integrated over one complete cycle. As the time integral of a current is a quantity of electricity, each of the integrals of current will be represented by $q$ with the same subscript as the corresponding current. Since the detector bridge is connected to the primary bridge during a part of the cycle only, the integrals of the current in the primary bridge will be considered only during that portion of the cycle when the detector bridge is connected to it. The time that each event in the cycle occurs will be designated as follows:

$t_{0}=$ zero time $=$ time circuit of primary bridge is closed $\left(C_{1}\right.$ makes contact), the detector bridge having been previously connected to the primary bridge.

$t_{1}=$ time detector bridge is disconnected $\left(C_{2}\right.$ breaks contact $)$,

$t_{2}=$ time circuit of outer bridge is opened ( $C_{1}$ breaks contact),

$t_{3}=$ time detector bridge is again connected $\left(C_{2}\right.$ makes contact),

Also

$t_{4}=$ end of cycle.

\section{$T=t_{4}-t_{0}=$ time of a complete cycle.}

The current $I_{3}$ is maintained only when the detector bridge is connected, which occurs in the intervals $t_{0}$ to $t_{1}$ and $t_{3}$ to $t_{4}$. In the interval $t_{3}$ to $t_{4}$ the circuit of the primary bridge is open, and $I_{3}$ will be zero provided the interval $t_{2}$ to $t_{3}$ has been sufficiently long to allow the currents of the primary bridge to become zero. Under this condition, the detector bridge is influenced only by the integral of $I_{3}$ during the interval $t_{0}$ to $t_{1}$. On the other hand, the current from the battery $e$ in the detector bridge and that resulting from the motion of the galvanometer coil are effective throughout the cycle.

The time $t_{0}$ was chosen so that at $t=0$, both $I_{1}$ and $I_{3}$ would be zero. Representing the values of $I_{1}$ and $I_{3}$ at time $t_{1}$ as $I_{1}\left(t_{1}\right)$ and $I_{3}\left(t_{1}\right)$ and at other times in a corresponding manner, the assumption will now be made that $I_{3}\left(t_{1}\right)=0, I_{1}\left(t_{1}\right)=I_{1}\left(t_{\infty}\right)$, and $I_{1}\left(t_{3}\right)=0$. The conditions under which these assumptions are valid will be discussed later. Integrating each of the eq 3 to 7 from 0 to $T$, but separating each integral into parts and retaining only those parts that are integrated over the times that the integrated result affects the galvanometer of the detector bridge, the following equations result:

$$
\begin{aligned}
B q_{1}+(B+P+R) q_{2}-R q_{3}-R q_{4} & =E t_{1} . \\
Q q_{1}-P Q_{2}-(h+k) q_{4}+k q_{5} & =-L I_{1}\left(t_{1}\right) . \\
S q_{1}-R q_{2}+(R+S) q_{3}+(R+S+h+k) q_{4} & =0 . \\
(f+g) q_{3}-(h+k) q_{4}+(k+f) q_{5} & =0 . \\
f q_{3}-k q_{4}+(b+f+k) q_{5} & =0 .
\end{aligned}
$$

These equations can be solved by determinants for $q_{3}$. In this solution, the minor by which $E t_{1}$ is multiplied vanishes, since $Q R=P S$. Then, using the equality $g k=f h$,

$$
q_{3}=\frac{R k L I_{1}\left(t_{1}\right)}{P(R+S)(f+k)+f(P+R)(h+k)} .
$$


But

$$
I_{1}\left(t_{1}\right)=I_{1}\left(t_{\infty}\right)=\frac{E R}{B(R+S)+S(P+R)}
$$

and by eq 2 ,

$$
q_{3}=\frac{e k T}{b(h+k)+h(f+k)} .
$$

Solving eq 13,14 , and 15 for $L$,

$$
L=T \cdot \frac{e}{E} \cdot \frac{[B(R+S)+S(P+R)][P(R+S)(f+k)+f(P+R)(h+k)]}{R^{2}[b(h+k)+h(f+k)]}
$$

If both bridges are "square," so that $P=R=S$ and $f=h=k$,

$$
L=T \cdot \frac{e}{E} \cdot \frac{4(B+P)(P+f)}{b+f}
$$

Equation 17 is useful in determining the most suitable resistances to be used in a given case, but it should not be used for precise computations, since the bridges are never exactly square.

The value of the inductance $L$, as given by eq 16 , is determined from the rate of rotation of the camshaft, the ratio of the two electromotive forces, and the resistances of the arms of the bridges. The rate of rotation can be maintained constant and can be accurately measured. The ratio of the electromotive forces can be accurately measured by means of a calibrated potentiometer. The resistances of all the arms of both bridges except the one containing the inductance in the primary bridge and the one containing the galvanometer in the detector bridge must be known. However, these excepted resistances are directly involved, since the derivation assumes that both primary and detector bridges are balanced for direct current. Hence, any change in either of these resistances between the time of balancing the two bridges with direct current, using the auxiliary galvanometer, and the time of balancing the dual bridge introduces an error in the computed value of $L$. With care, any error on this account can be made negligible. The precision with which the resistance $B$ of the battery arm of the primary bridge can be measured will often determine the accuracy that can be attained by this method.

The conditions for determining that, within a specified error, $I_{3}\left(t_{1}\right)=0, I_{1}\left(t_{1}\right)=I_{1}\left(t_{\infty}\right)$, and $I_{1}\left(t_{3}\right)=0$ can be obtained by solving the eq 3 to 7 for the currents $I_{1}$ and $I_{3}$, and determining their values at the indicated times. The purpose is to set limits both to the values of the resistances that may be used and to the length of the period that may be employed when a given inductance is being measured. Since high accuracy is not required in setting these limits, the equations can be simplified by neglecting the very small electromotive force introduced by the motion of the galvanometer coil. Also, since the arrangement of the bridge is such that $I_{3}(t)$ approaches its final value more slowly than $I_{1}(t)$, the bridge constants that make $I_{3}\left(t_{1}\right) \cong 0$ will certainly make $I_{1}\left(t_{1}\right) \cong I_{1}\left(t_{\infty}\right)$. Also the bridge arrangement is such that $I_{1}$ dies down somewhat more rapidly than it builds up. Hence all conditions will be satisfied if $I_{3}\left(t_{1}\right)$ is sufficiently near zero. 
Rearranging eq 3 to 7 , solving by determinants for $I_{3}$, and noting that the application of the symbolic operator, $D_{t}$, to the electromotive force $E$, or to any of the resistances, gives zero, the value of $I_{3}$ can be obtained from the equation

$$
\Delta I_{3}=0,
$$

where $\Delta$ is the determinant formed by the coefficients of the currents in eq 3 to 7 . Expanding this determinant,

where

$$
\left[\alpha l L D_{\mathbf{t}}^{2}+(\beta L+\gamma l) D_{t}+\delta\right] I_{3}=0,
$$

$$
\begin{gathered}
\alpha=[(B+P)(R+S+h)+R(S+h)][b+f+k]+k(b+f)(B+P+R) . \\
\beta=\frac{1}{k}\{(f+k)[B(R+S)+S R+S P+P R]+f(h+k)(B+P+R)\} \\
\gamma=\frac{1}{R}\{B(R+S)+S(P+R)\} \\
\qquad[(h+k)+h(f+k)\} . \\
\delta=\frac{1}{R k}[B(R+S)+S(P+R)][b(h+k)+h(f+k)] \\
\qquad P(R+S)(f+k)+f(P+R)(h+k)] .
\end{gathered}
$$

Solving the differential eq 19 ,

where

$$
\begin{gathered}
I_{3}=A_{1} \epsilon^{-(\lambda-\sigma) t}+A_{2} \epsilon^{-(\lambda+\sigma) t}, \\
\lambda=\frac{\beta L+\gamma l}{2 \alpha l L}, \sigma=+\frac{\sqrt{(\beta L+\gamma l)^{2}-4 \alpha \delta l L}}{2 \alpha l L}
\end{gathered}
$$

and $A_{1}$ and $A_{2}$ are the constants of integration. Equation 25 shows that $\sigma$ is always smaller than $\lambda$, but is a real number for all practical bridge arrangements.

When $t=0, I_{3}=0$, so that $A_{2}=-A_{1}$,

$$
I_{3}=A_{1}\left[\epsilon^{-(\lambda-\sigma) t}-\epsilon^{-(\lambda+\sigma) t}\right] .
$$

This equation shows that $I_{3}$ may be considered as the difference of two currents, each of which has the value $A_{1}$ when $t=0$, and botb of which decrease to zero at infinite time. However, the current for which the exponent of $\epsilon$ is the smaller decreases less rapidly than the other current, so that the difference goes through a maximum and decreases towards zero. The relationship of these two currents to each other and to their difference is shown in figure 2. In this figure the value of $A_{1}$ is taken as unity, and the value of $\lambda+\sigma$ only twice that of $\lambda-\sigma$, which is a much smaller difference than would be found in an actual bridge. The values of $y$ correspond to the current and its components. However, even in this case, the difference curve rapidly approaches the value of the curve with the smaller exponent.

The total quantity of electricity which passes through the ballistic galvanometer, and which produces the torque on its coil, is the integral of $I_{3}$, as given in eq 25. When the time is indefinitely long,

$$
\int_{0}^{\infty} I_{3} d t=A_{1} \int_{0}^{\infty} \epsilon^{-(\lambda-\sigma) t} d t-A_{1} \int_{0}^{\infty} \epsilon^{-(\lambda+\sigma) t} d t=A_{1}\left[\frac{2 \sigma}{\lambda^{2}-\sigma^{2}}\right] .
$$


The quantity which actually passes through the galvanometer when the circuit is opened at time $t_{1}$ is less than the above amount by the integral

$$
\int_{t_{1}}^{\infty} I_{3} d t=A_{1}\left[\frac{\epsilon^{-(\lambda-\sigma) t_{1}}}{\lambda-\sigma}-\frac{\epsilon^{-(\lambda+\sigma) t_{1}}}{\lambda+\sigma}\right]
$$

Hence the ratio, $\zeta$, of the deficiency in quantity at time $t_{1}$ to the quantity at infinite time is

$$
\zeta=\frac{\lambda+\sigma}{2 \sigma} \epsilon^{-(\lambda-\sigma) t_{1}}-\frac{\lambda-\sigma}{2 \sigma} \epsilon^{-(\lambda+\sigma) t_{1}} .
$$

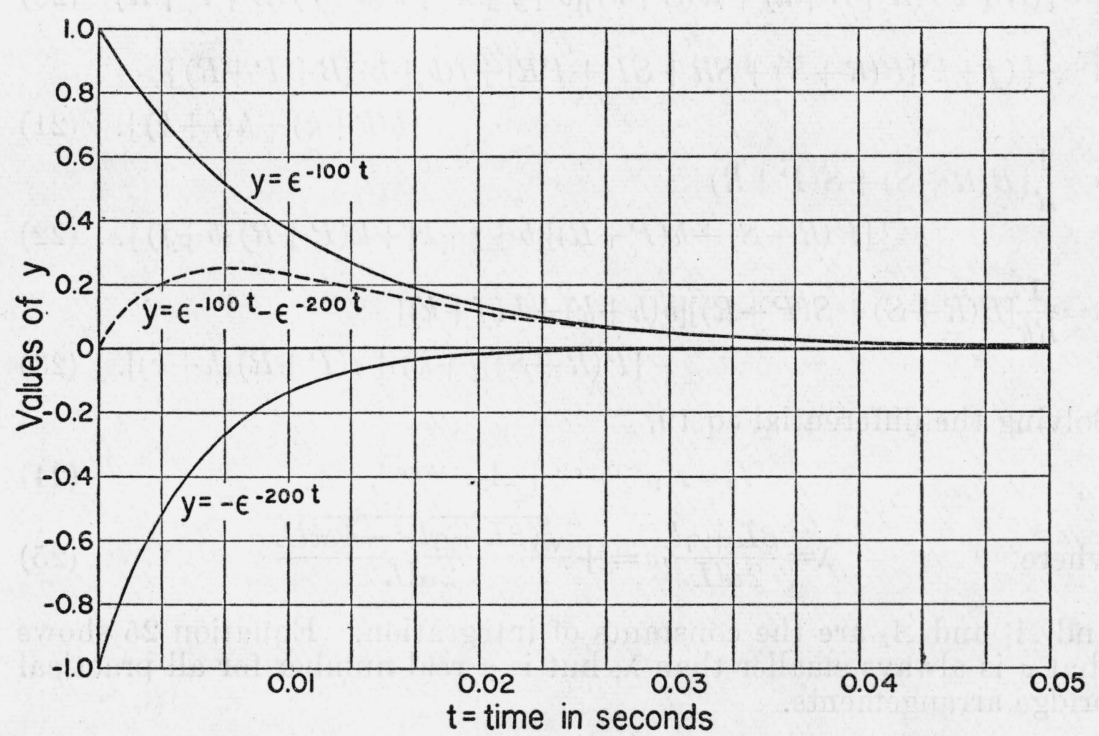

FIGURE 2.-Curves of exponential functions to show the difference of two functions with negative exponents when one exponent has twice the value of the other.

This equation can be used to compute, for any given bridge arrangement, the error that will be introduced by allowing only the time $t_{1}$ for the currents to reach a steady state. The reverse process of determining the maximum value of $t_{1}$ when the error is to be no greater than $\zeta$, is not readily carried out. However, the second term of eq 29 decreases so much more rapidly than the first term that at time $t_{1}$ only the first term needs to be considered. This decrease makes the second term negligibly small in a shorter interval than is shown by the curve of $\epsilon^{-(\lambda+\sigma) t}$ in figure 2 , since the coefficient of the second term of eq 29 is smaller than the coefficient of the first term, whereas in the plotted curves, both terms have the same coefficient. Hence the shortest time, $t_{1}$, during which both switches $C_{1}$ and $C_{2}$ must be closed when an accuracy of $\zeta$ is required, can be determined from the equation

$$
\zeta=\frac{\lambda+\sigma}{2 \sigma} \epsilon^{-(\lambda-\sigma) t_{1}}
$$

This equation is used onl $\mathrm{y}$ to set a lower limit for $t_{1}$, and any approximation that makes this limit lower than that given by eq 30 will not 
affect the accuracy of a determination of the inductance $L$, although it may increase the difficulty of choosing suitable constants for the dual bridge. Hence $(\lambda+\sigma) / 2 \sigma$ may be taken as unity, since it is always larger than that, and such an error would make $t_{1}$ too small. Using this approximation, and taking the natural logarithm of both sides of eq 30 ,

$$
(\lambda-\sigma) t_{1}=-\ln \zeta=-2.3 \log \zeta .
$$

The following example indicates a suitable procedure for computing the limiting value for the period, $T$, of the pulsating current when the bridge constants have been selected. Assume that both the primary and detector bridges are square, that the resistances of the detector bridge are at least 10 times greater than those of the primary bridge, and that the inductances are of the same order of magnitude. Then $P=Q=R=S ; f=g=h=k=a P$; and $l=c L$, where $a$ is greater than 10 and $c$ is less than one-tenth of $a$. Assume also that $B=0$, that $b$ is very large relative to any other resistance in the bridges, that $T=2 t_{1}$, and that the required accuracy is 1 part in a million $\left(\zeta=10^{-6}\right)$. Substituting these values in eq 20 to 25 to obtain values of $\lambda$ and $\sigma$, using these values in eq 31, and neglecting some terms that introduce an error of less than 10 percent in the value of $T$,

$$
T \geqslant \frac{14 L}{P} \text {. }
$$

If $L=0.1$ henry and $P=100 \mathrm{ohms}$, no error will be introduced in the value of $L$ because of the shortness of the cycle if $T$ is greater than $0.014 \mathrm{sec}$ (the frequency of the pulsating current less than $70 \mathrm{c} / \mathrm{sec}$ ).

\section{EXPERIMENTAL TEST OF THE METHOD ${ }^{3}$}

The experimental test of the method was undertaken before the complete theory had been developed in order to determine whether there were serious defects in it that could only be discovered by such a test. The work showed that the method had possibilities for use in precision measurements. The complete theory given in the preceding pages was then developed. However, the formula for computing the inductance was not changed by the more complete analysis.

\section{THE BRIDGE}

The resistance of each of the arms $(P, Q, R, S)$ of the primary bridge was $300 \mathrm{ohms}$, and that of earh of the arms $(f, g, h, k)$ of the detector bridge was $10,000 \mathrm{ohms}$. The resistor $b$ was a dial box which could be varied from $1 \mathrm{ohm}$ to $10,000 \mathrm{ohms}$. All of these resistors were guaranteed by the makers to be accurate to $1 / 20$ percent, but they were not calibrated during the experimental work. Variable resistors in the $Q$ and $g$ arms permitted the primary and detector bridges to be balanced to closer than 1 part in 10,000 when the auxiliary galvanometer was employed.

${ }_{3}$ The junior author started the experimental work at the National Bureau of Standards in the summer of 1930. He made an entirely new setup in the Physics Department at the University of Nevada, where the work was carried to completion. The role of second observer to control the speed of the motor generator set was taken by one or another of the children of the junior author. 


\section{THE GALVANOMETERS}

The galvanometer used in the detector bridge had a period of 15 sec. This instrument was adjusted until it integrated correctly the current passing through it. ${ }^{4}$ In order to test the effect of the inductance of the galvanometer, additional inductances varying from 50 to 550 $\mathrm{mh}$ were inserted in series with the galvanometer, but no change in the balance of the bridge could be observed. The auxiliary galvanometer, which was provided with a suitable Ayrton shunt, had a sensitivity of $4.5 \times 10^{-9} \mathrm{amp} / \mathrm{mm}$ at a distance of $1 \mathrm{~m}$.

\section{THE BATTERIES}

Battery $e$ in the detector bridge was a dry cell, and battery $E$ in the primary bridge was a storage battery. The electromotive forces

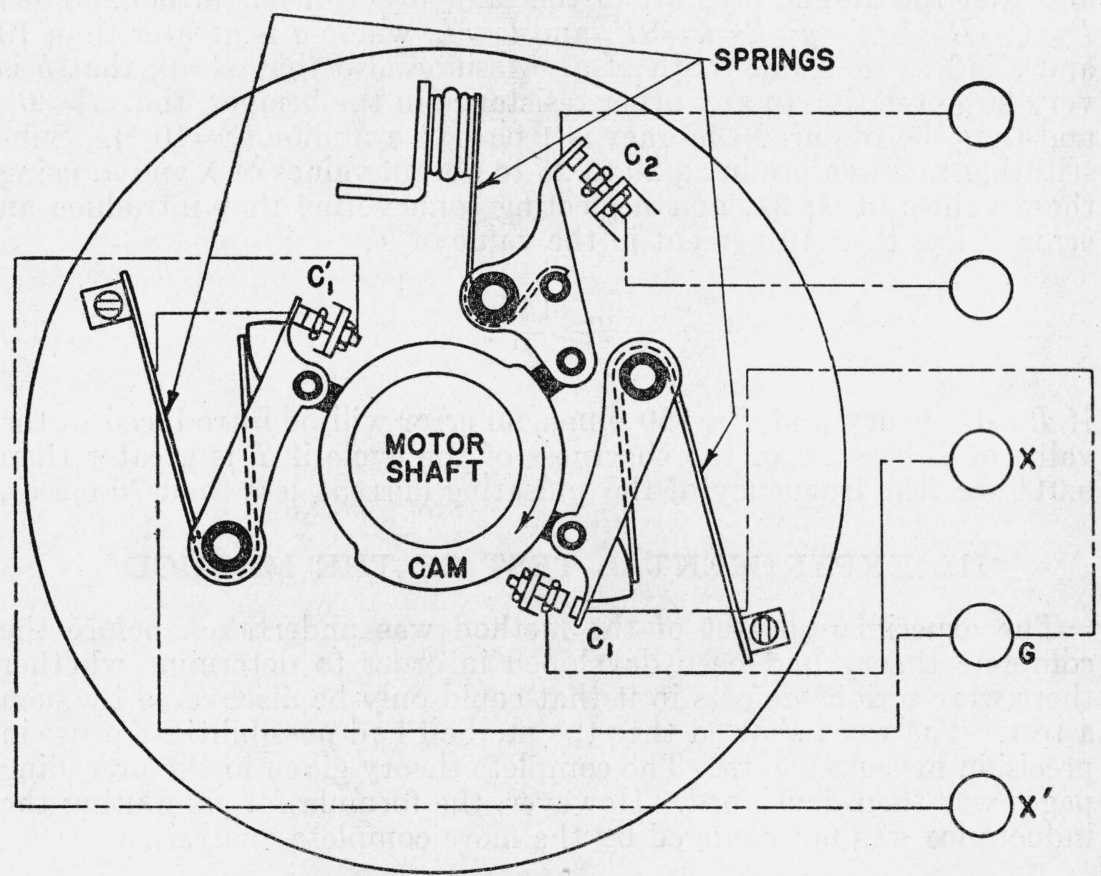

FIGURE 3.-Diagram of motor-operated switches for the dual bridge.

Either switch $C_{1}$ or $C^{\prime}{ }_{1}$ may be used for making and breaking the current in the primary bridge; the switch $C_{2}$ opens and closes the connection between the primary and detector bridges. If $C_{1}$ is used in the primary bridge, the unbalance of the primary bridge on closing the circuit is measured by the detector bridge; if $C^{\prime}{ }_{1}$ is used, the balance on opening is measured.

of $e$ and $E$ were compared by means of a potentiometer. The resistance of the storage battery was determined by measuring, with a potentiometer, the electromotive force on open circuit and then the fall of potential through a known resistance connected directly across its terminals. The difference between these two values gave the drop in potential through the battery when a known current was passing through it, from which the internal resistance of the primary battery was computed.

- See p. 507 of paper by Curtis and Moon, BS Sci. Pap. 22 (1927) S564. 


\section{THE CAM-OPERATED SWITCHES}

The device finally adopted for opening and closing the circuits (see fig. 3) consisted essentially of the contact maker of a Liberty airplane motor. This device was mounted upon the free end of the shaft of a motor generator set and was adjusted so that it was free from "chattering" when the motor was driven at relatively low speed. Oscilloscope tests showed that the rise and fall of current due to the induced electromotive force in $Q$ gave a smooth and apparently complete logarithmic curve. The circuit through switch $C_{2}$, which joined the secondary bridge to the primary, was closed about one-fifth of a revolution of the motor before the closing of the circuit through switch $C_{1}$, which joined battery $B$ to the primary bridge. The circuits through the two switches then remained closed for slightly less than onequarter of a revolution of the motor, at which time the circuit to the detector bridge was opened while the circuit of the primary bridge remained closed for slightly more than one-sixth of a revolution of the motor. During the remainder of the revolution of the cam, both bridge circuits were open. Thus the inductance of the coil was measured by the effect produced when the current through the inductor was rising from zero to its maximum value. By changing one lead to the primary bridge from $X$ to $X^{\prime}$ and reversing the leads connecting the secondary bridge to $C_{2}$, the inductance of the coil could be measured by the effect produced when the current through the inductor was falling from its maximum value to zero.

\section{CONTROL AND MEASUREMENT OF THE FREQUENCY OF THE PULSATING CURRENT}

The frequency of the pulsating current was determined by the speed of a motor generator on the shaft of which was mounted the cam for opening and closing the circuits. The speed was maintained constant by an observer who so varied the pressure of his finger on the rim of a flywheel attached to the shaft that a pointer rotating with the shaft appeared to remain stationary when illuminated by a neon lamp that was flashed by contacts on a tuning fork. The speed was measured by a chronograph ${ }^{5}$ which was geared to the shaft. The time intervals for this chronograph depended on a standard clock that was frequently calibrated by means of radio signals. The average speed for any one observation was not in error by as much as 1 part in 10,000.

\section{PROCEDURE IN MAKING OBSERVATIONS}

In making a set of observations, one observer controlled the speed of the motor generator set and obtained a record of it on the chronograph while a second observer made the necessary bridge adjustments. During the 2 or 3 minutes which were required to make a record on the chronograph that was sufficiently long to permit an accurate measurement of the speed, the second observer would make several cycles of adjustments of the bridges. In each cycle the primary bridge was first connected directly to the battery and balanced by using the auxiliary galvanometer and varying $Q$, then the secondary bridge was connected to its battery and balanced by using the same galvanometer and varying $g$, and finally the dual bridge was connected to the two

\footnotetext{
8 Phil. Mag. 155, 518 (1865).
} 
batteries through the cam-operated switches and balanced by varying $b$ until the ballistic galvanometer in the detector bridge showed no deflection. When a cycle was reached in which no change from the preceding cycle was required in any of the resistances $Q, g$, or $b$, the dual bridge was considered balanced. The electromotive forces of the cells were then immediately compared by a potentiometer.

\section{RESULTS}

The results given in table 1 were obtained on two fixed standards of self inductance having nominal values of 1 henry and 0.1 henry, on the 1-henry inductor and an 0.01-henry inductor connected in series, and on a group of the three inductors mentioned above connected in series. In the series connections, the coils were so arranged that there was no mutual inductance between them. The measurements with the dual bridge were made at the University of Nevada. The alternating-current values were obtained at the National Bureau of Standards, by measuring each inductor separately, using a frequency of 60 c/sec. The values obtained for aiviven inductor with the dual bridge differ among themselves by as much as 3 parts in 1,000, which is the result of experimental error. The average values for the inductances having a value of about 1 henry, for which the setup was particularly adapted, differ from the alternating-current value by only 2 or 3 parts in 10,000. This difference might be the result of instability in the inductors themselves. Hence there is no indication that a systematic error occurred in the measurements with the dual bridge.

TABLE 1.-Results obtained with the dual bridge, on four different inductances, compared with their alternating-current values

\begin{tabular}{|c|c|c|c|c|}
\hline \multicolumn{4}{|c|}{ Measurements with dual bridge } & \\
\hline $\begin{array}{l}\text { Number of } \\
\text { makes and } \\
\text { breaks per } \\
\text { second }\end{array}$ & $\begin{array}{c}\text { Measured } \\
\text { inductance }\end{array}$ & $\begin{array}{l}\text { Number of } \\
\text { makes and } \\
\text { breaks per } \\
\text { second }\end{array}$ & $\begin{array}{l}\text { Measured } \\
\text { inductance }\end{array}$ & $\begin{array}{l}\text { Alternating } \\
\text { current } \\
\text { inductance }\end{array}$ \\
\hline \multicolumn{5}{|c|}{ 100-mh INDUCTOR } \\
\hline \multirow[t]{2}{*}{$\begin{array}{l}9.0883 \\
9.0897\end{array}$} & $\begin{array}{l}m h \\
99.89 \\
99.80\end{array}$ & $\begin{array}{r}10.0006 \\
9.9974\end{array}$ & $\begin{array}{c}m h \\
100.13 \\
100.11\end{array}$ & $\begin{array}{c}m h \\
-4\end{array}$ \\
\hline & & Mean ...... & 99.98 & 100.08 \\
\hline \multicolumn{5}{|c|}{ 1-h INDUCTOR } \\
\hline $\begin{array}{r}14.2980 \\
14.2977 \\
14.2856 \\
11.1118 \\
9.0967 \\
9.0941 \\
11.1403 \\
9.9976 \\
10.0165 \\
8.3248 \\
12.4970 \\
9.1020 \\
14.2980 \\
10.0223 \\
10.0204\end{array}$ & $\begin{array}{l}m h \\
994.8 \\
994.7 \\
994.6 \\
994.0 \\
995.0 \\
994.4 \\
994.6 \\
995.2 \\
995.8 \\
995.0 \\
994.2 \\
994.9 \\
994.8 \\
996.1 \\
995.6\end{array}$ & $\begin{array}{r}10.0196 \\
10.0169 \\
10.0238 \\
10.0253 \\
10.0242 \\
10.0228 \\
10.0216 \\
10.0262 \\
10.0242 \\
8.3467 \\
8.3472 \\
8.3424 \\
5.8908 \\
4.1753 \\
4.5551\end{array}$ & $\begin{array}{l}m h \\
996.0 \\
995.2 \\
994.1 \\
994.0 \\
994.0 \\
994.8 \\
995.4 \\
995.6 \\
996.0 \\
994.7 \\
994.2 \\
994.1 \\
994.1 \\
995.1 \\
995.9\end{array}$ & $\begin{array}{c}{ }^{m h} \\
\\
\end{array}$ \\
\hline 10.0204 & & Mean..... & 994.9 & 994.7 \\
\hline
\end{tabular}


TABLE 1.-Results obtained with the dual bridge, on four different inductances, compared with their alternating-current values - Continued

\begin{tabular}{|c|c|c|c|c|}
\hline \multicolumn{4}{|c|}{ Measurements with dual bridge } & \multirow[b]{2}{*}{$\begin{array}{l}\text { Alternating } \\
\text { current } \\
\text { inductance }\end{array}$} \\
\hline $\begin{array}{l}\text { Number of } \\
\text { makes and } \\
\text { breaks per } \\
\text { second }\end{array}$ & $\begin{array}{c}\text { Measured } \\
\text { inductance }\end{array}$ & $\begin{array}{l}\text { Number of } \\
\text { makes and } \\
\text { breaks per } \\
\text { second }\end{array}$ & $\begin{array}{l}\text { Measured } \\
\text { inductance }\end{array}$ & \\
\hline \multicolumn{5}{|c|}{ 1-h INDUCTOR IN SERIES WITH A 10 -mh INDUCTOR } \\
\hline \multirow[t]{2}{*}{$\begin{array}{r}14.3017 \\
14.3002 \\
14.3002 \\
14.2906 \\
16.6485 \\
11.0970 \\
9.9889\end{array}$} & $\begin{array}{c}m h \\
1004.3 \\
1004.0 \\
1004.0 \\
1004.8 \\
1004.6 \\
1005.3 \\
1005.2\end{array}$ & $\begin{array}{r}9.9950 \\
8.3330 \\
8.3341 \\
9.1128 \\
11.1373 \\
9.9972 \\
\end{array}$ & $\begin{array}{c}m h \\
1004.9 \\
1005.1 \\
1005.1 \\
1005.4 \\
1005.6 \\
1005.0\end{array}$ & $\begin{array}{c}m_{h} \\
\\
\end{array}$ \\
\hline & & Mean .... & 1004. 9 & 1004.7 \\
\hline \multicolumn{5}{|c|}{ 1-h INDUCTOR, 100-mh INDUCTOR, AND 10-mh INDUCTOR, ALL IN SERIES } \\
\hline $\begin{array}{r}10.0006 \\
10.0006 \\
9.9927 \\
10.0035 \\
11.1075\end{array}$ & $\begin{array}{c}m h \\
1106.4 \\
1106.4 \\
1106.6 \\
1103.6 \\
1104.6\end{array}$ & $\begin{array}{l}\text { 11. } 1041 \\
11.1041 \\
11.1073 \\
11.1073 \\
11.1136\end{array}$ & $\begin{array}{c}m h \\
1105.0 \\
1105.3 \\
1104.8 \\
1104.5 \\
1103.9\end{array}$ & $\begin{array}{c}m h \\
\end{array}$ \\
\hline 11.1075 & & Mean..... & 1105.1 & 1104.8 \\
\hline
\end{tabular}

\section{SUMMARY AND CONCLUSION}

A new method for the absolute measurement of inductance, which employs a dual bridge, has been described. The theory of the bridge has been developed showing that the inductance can be determined in terms of certain constants of the bridge, which can be accurately measured. In developing the theory, factors which might introduce an error of 1 part in a million in measuring an inductance of 0.1 henry or larger have been considered.

The inductances of several coils have been measured by this method, giving results, within experimental error, which agree with those obtained by an alternating-current method. The experimental determinations were undertaken to determine if there were any unsurmountable difficulties in applying the method and were not carried out in a manner to obtain high precision. Both the theory and the results of the measurements indicate that the method is capable of giving precise results.

Washington, April 18, 1940. 